knowledge score among trainees $(\mathrm{p}<0.05)$. The project has also provided a platform for sharing experiences and maintaining partnerships with regional and international institutes in addition to provision of technical support for newly established RECs.

Conclusion We believe that the array of activities conducted through this project had enhanced the governance, coordination, feasibility and, efficiency of the ethical review system in Sudan.

\section{OC 8717 TRANSFER OF LEADERSHIP, WHAT DO WE NEED?}

Stellah Mpagama. Kibong'oto Infectious Diseases Hospital, Kilimanjaro, Tanzania

\subsection{6/bmjgh-2019-EDC.41}

Background The Pan African Consortium for the Evaluation of Anti-Tuberculosis Antibiotics (PanACEA) was designed to build clinical tuberculosis (TB) trial capacity whilst conducting clinical trials investigating novel and existing agents to shorten and simplify TB treatment. One of the objectives of the programme is to foster leadership development in sub-Saharan TB-endemic countries to move leadership to African partners in the PanACEA research programmes.

Methods and results In PanACEA 1 the participation of the sites on the consortium board was important to foster leadership development. African investigators now make up a large part of the consortium leadership and are actively developing new concepts. Delegates of the sites visited the annual PanACEA General Assembly meetings, where they could gain knowledge, actively participate in the meetings and discussions and network with others from the TB research community. Various sites participated at TB research community conferences (e.g. CROI, Lung Health Meeting) where PanACEA members gave presentations and could collaborate with other TB trial networks.

In PanACEA 2 all clinical trials are co-led by a European established researcher and an African Principal Investigator (PI), to ensure that African scientists are trained and mentored to lead in every aspect essential to clinical trial delivery, from trial and data management to statistical analysis and trial design, and from financial management to laboratory science. The capacity development cores, which serve as operational support for all PanACEA studies and provide high level oversight, also use the European and African countersystem, including senior and junior co-chairs among the African trial sites.

An example of leadership transfer is a large EDCTP application for the development of a new compound in MDR TB (FACE-MDR-TB) in which Stellah Mpagama is the lead applicant.

Conclusion The PanACEA consortium has actively facilitated a transfer of leadership programme which may be successful in future consortia.

\section{OC 8718 WHAT DID WE LEARN FROM PANACEA 1 CLINICAL TRIALS?}

Nyanda E Ntinginya. NIMR - Mbeya Medical Research Centre, Tanzania

10.1136/bmjgh-2019-EDC.42

Background The Pan African Consortium for the Evaluation of Anti-Tuberculosis Antibiotics (PanACEA) was designed to build clinical tuberculosis (TB) trial capacity whilst conducting clinical trials on novel and existing agents to shorten and simplify TB treatment. One of the objectives was to conduct, mentor and monitor observational and clinical studies at sites in 6 Sub-Saharan TB-endemic countries (Gabon, Kenya, South Africa, Tanzania, Uganda and Zambia)

Methods Learning through experience. All centres in the 6 countries self-assessed their requirements for capacity development in the following fields: a) clinical staff availability and experience; b) TB laboratory infrastructure and staff; c) safety laboratory infrastructure and staff; d) clinical site facilities and equipment; e) pharmacy facilities and staff; IT facilities; and f) overall training needs of site personnel.

Results From March 2011 - June 2014, we conducted four epidemiological studies (characterising TB patient populations in preparation for future studies) and five phase II studies (GCP standard intervention trials).

By working together in epidemiological and clinical trials, the sites identified their needs for resources and training as well as developing capabilities to perform independent largescale TB clinical trials beyond PanACEA-initiated trials. Through the ReMoxTB study, for example, laboratories were brought to an international standard for safety and mycobacterial expertise. Furthermore, through developing skill-sets related to EBA studies, sites have since then attracted other sponsors for further studies.

Sites could be mentored to perform GCP-compliant clinical TB trials that is built on sound physical infrastructure, training and strong on-site leadership.

Conclusion The learning-by-doing approach meant that staff could be trained whilst acquiring new core competencies and revealing operational gaps. Our experience of conducting TB trials within an environment of mentoring, networking and training has provided a platform for establishing future sustainable research centres that has capacities to conduct highly regulated studies.

\section{OC 8721 WANECAM II - A CLINICAL TRIAL PROGRAMME TO ASSESS SAFETY, EFFICACY AND TRANSMISSION- BLOCKING PROPERTIES OF A NEW ANTIMALARIAL KAF156 (GANAPLACIDE) IN UNCOMPLICATED MALARIA IN WEST AND CENTRAL AFRICA}

${ }^{1}$ Abdoulaye Djimde ${ }^{*}$, on behalf of the WANECAM II consortium, ${ }^{6}$ Martin P Grobusch, ${ }^{4}$ Rella Zoleko Manego, ${ }^{4}$ Ghyslain Mombo-Ngoma, ${ }^{7}$ Stephane Picot, ${ }^{1}$ Issaka Sagara, ${ }^{8}$ Colin Sutherland, ${ }^{1}$ Aminatou Kone, ${ }^{1}$ Ogobara K Doumbot, ${ }^{9}$ Jose Pedro Gil, ${ }^{9}$ Anders Björkman, ${ }^{12}$ Steffen Borrmann, ${ }^{3}$ Issiaka Soulama, ${ }^{1}$ Bakary Fofana, ${ }^{11}$ Stephan Duparc, ${ }^{1}$ Alassane Dicko, ${ }^{10}$ David Hughes, ${ }^{10}$ Cornelis Winnips, ${ }^{2}$ Sodiomon B Sirima, ${ }^{5}$ Eric Adehossi, ${ }^{13}$ Jean-Bosco Ouedraogo, 'Laurent Dembele, ${ }^{3}$ Issaka Zongo, ${ }^{11}$ Sophie Biguenet, ${ }^{2}$ Edithe Ilboudo-Sanogo, ${ }^{3}$ Aminata Fofana. ${ }^{1}$ Université des Sciences, des Techniques et des Technologies de Bamako (USTTB), Mali; ${ }^{2}$ Groupe de Recherche Action en Santé (GRAS), Burkina Faso; ${ }^{3}$ Institut des Sciences et Techniques (INSTech), Ouagadougou Burkina Faso; ${ }^{4}$ Centre de Recherches Médicales en Lambaréné (CERMEL), Libreville, Gabon; ${ }^{5}$ University Abdou Moumouni of Niamey, Niger; ${ }^{6}$ Academic Medical Center (AMC), Amsterdam, The Netherlands; ${ }^{7}$ University Lyon 1, France; ${ }^{8}$ London School of Hygiene and Tropical Medicine, UK; ${ }^{9}$ Karolinska Institute (KI), Stockholm, Sweden; ${ }^{10}$ Novartis Pharma $A G$, Basel, Switzerland; " ${ }^{11}$ Medicines for Malaria Venture (MMV), Geneva, Switzerland; ${ }^{12}$ Eberhard Karls Universität Tübingen, Germany

\subsection{6/bmjgh-2019-EDC.43}

Background Despite major progress in the past decade, malaria remains a major public health problem in sub-Saharan Africa. West and Central Africa account for nearly $2 / 3$ of the burden currently attributable to falciparum malaria. Artemisinin-based 